\title{
Correction and comment on "thermal conductance of nanofluids: is the controversy over?"
}

\author{
S. M. Sohel Murshed
}

Received: 7 September 2008/Accepted: 22 October 2008/Published online: 25 November 2008

(C) Springer Science+Business Media B.V. 2008

In the October 2008 (No. 7, pp. 1089-1097) issue of the Journal of Nanoparticle Research, Keblinski et al (2008) published an article entitled "Thermal conductance of nanofluids: is the controversy over?". Besides brief review of heat transfer mechanisms and controversy on nanofluids, the article also brings out an effective medium theory-based classical model (Hashin and Shtrikman 1962) to predict the effective thermal conductivity of nanofluids. This is a very interesting article. It intercalates a mechanism (aggregation of particles), which was previously considered ineffective (Keblinski et al. 2002), and denies most of the highlighted mechanisms such as particle size, Brownian motion, micro-convection, and dispersion (e.g., Jang and Choi 2004; Chon et al. 2005; Koo and Kleinstreuer 2004; Prasher et al. 2005; Hong and Yang 2005) for the enhanced thermal conductivity of nanofluids. Unfortunately, the article contains two sets of wrong thermal conductivity data.

Under Section "Discussion" two graphs in Fig. 1 on p. 6 of Keblinski et al.'s (2008) article, the authors surprisingly presented thermal diffusivity results for

See also doi:10.1007/s11051-007-9352-1.

S. M. Sohel Murshed $(\square)$

Department of Mechanical, Materials, and Aerospace Engineering, University of Central Florida, 4000 Central Florida Blvd, Orlando, FL 32816, USA

e-mail: smurshed@mail.ucf.edu
$\mathrm{Al} / \mathrm{EG}$ (ethylene glycol) and Al/engine oil-based nanofluids obtained from literature (i.e., Murshed et al. (2006)) as the thermal conductivity data of these nanofluids. These must be corrected. There are also typos in author's name of the cited article spotted at the top of those two graphs in Fig. 1.

According to the argumentation of Keblinski et al (2008), if aggregation of particles is responsible for the reported anomalously high thermal conductivity of nanofluids, the use of surfactant and sonication, which works against agglomeration or clustering and improve dispersion of nanoparticles in the base fluid resulting in higher thermal conductivity (Xie et al. 2003; Murshed et al. 2005; Hong and Yang 2005; Yu et al. 2008), are insignificant. Some researchers (e.g., Karthikeyan et al. 2008; Hong and Yang 2005, Hong et al. 2005) also showed that the effective thermal conductivity of nanofluids decreases (or increases) with increasing elapsed time (or sonication time) due to clustering of nanoparticles, which increases with elapsed time and decreases with sonication time. In addition, it is so far undisputed that the smaller the particle size, the higher the effective thermal conductivity of nanofluids (e.g., Kumar et al. 2004; Jang and Choi 2004; Chon et al. 2005; Yu et al. 2008). These aforementioned issues (use of surfactant and sonication, elapsed time of the sample fluid, and particle size etc.) are generally contrary to the aggregation argumentation provided in the article of Keblinski et al (2008) to explain the enhanced thermal conductivity of nanofluids. 
Keblinski et al. (2008) did not provide any thermal conductivity data for the base fluids and the particle materials used in calculating the effective thermal conductivities of those nanofluids by Hashin-Shtrikman's (H-S) model (Hashin and Shtrikman 1962). $\mathrm{H}-\mathrm{S}$ model was also justified for $\mathrm{TiO}_{2} /$ water-based nanofluids [Fig. 1 on p. 5 in Keblinski et al. (2008)] and the data were obtained from an article of Murshed et al. (2005). However, by using correct values of thermal conductivities of $\mathrm{TiO}_{2}$ nanoparticle and water, which are $8.40 \mathrm{~W} / \mathrm{m} \cdot \mathrm{k}$ (Masuda et al. 1993) and $0.607 \mathrm{~W} / \mathrm{m} \cdot \mathrm{k}$ (Kaviany 2002), respectively, H-S upper bound model gives fairly lower results than the results reported in Fig. 1 (Figure for $\mathrm{TiO}_{2}$-water). Furthermore, Zhang et al.'s (2006) effective thermal conductivity data for this nanofluid which was measured at $10^{\circ} \mathrm{C}$ (NOT at room temperature) was provided in the same figure.

Nevertheless, more systematic and careful investigations are needed to resolve the controversy over the mechanism of the enhanced thermal conductivity of nanofluids as well as to minimize the discrepancies in the results from different research groups.

\section{References}

Chon CH, Kihm KD, Lee SP, Choi SUS (2005) Empirical correlation finding the role of temperature and particle size for nanofluid $\left(\mathrm{Al}_{2} \mathrm{O}_{3}\right)$ thermal conductivity enhancement. Appl Phys Lett 87:153107-1-153107-3

Hashin Z, Shtrikman S (1962) A variational approach to the theory of the effective magnetic permeability of multiphase materials. J Appl Phys 33:3125-3131

Hong TK, Yang HS (2005) Nanoparticle-dispersion-dependent thermal conductivity in nanofluid. J Korean Phys Soc 47:S321-S324

Hong TK, Yang HS, Choi CJ (2005) Study of the enhanced thermal conductivity of $\mathrm{Fe}$ nanofluids. J Appl Phys 97:064311-1-064311-4
Jang SP, Choi SUS (2004) Role of Brownian motion in the enhanced thermal conductivity of nanofluids. Appl Phys Lett 84:4316-4318

Karthikeyan NR, Philip J, Raj B (2008) Effect of clustering on the thermal conductivity of nanofluids. Mater Chem Phys 109:50-55

Kaviany M (2002) Principles of heat transfer. Wiley, New York

Keblinski P, Phillpot SR, Choi SUS, Eastman JA (2002) Mechanisms of heat flow in suspensions of nano-sized particles (nanofluids). Int J Heat Mass Transf 45:855-863

Keblinski P, Prasher R, Eapen J (2008) Thermal conductance of nanofluids: is the controversy over? J Nanopart Res 10:1089-1097

Koo J, Kleinstreuer C (2004) A new thermal conductivity model for nanofluids. J Nanopart Res 6:577-588

Kumar DH, Patel HE, Kumar VRR, Sundararajan T, Pradeep T, Das SK (2004) Model for heat conduction in nanofluids. Phys Rev Lett 93:4301-4304

Masuda H, Ebata A, Teramae K, Hishinuma N (1993) Alteration of thermal conductivity and viscosity of liquid by dispersing ultra-fine particles (dispersion of $\mathrm{Al}_{2} \mathrm{O}_{3}, \mathrm{SiO}_{2}$, and $\mathrm{TiO}_{2}$ ultra-fine particles). Netsu Bussei (Japan) 7:227-233

Murshed SMS, Leong KC, Yang C (2005) Enhanced thermal conductivity of $\mathrm{TiO}_{2}$-water based nanofluids. Int J Therm Sci 44:367-373

Murshed SMS, Leong KC, Yang C (2006) Determination of the effective thermal diffusivity of nanofluids by the double hot-wire technique. J Phys D Appl Phys 39:53165322

Prasher R, Bhattacharya P, Phelan PE (2005) Thermal conductivity of nanoscale colloidal solutions (nanofluids). Phys Rev Lett 94:025901-1-025901-4

Xie H, Lee H, Youn W, Choi M (2003) Nanofluids containing multiwalled carbon nanotubes and their enhanced thermal conductivities. J Appl Phys 94:4967-4971

Yu W, France DM, Routbort JL, Choi SUS (2008) Review and comparison of nanofluid thermal conductivity and heat transfer enhancements. Heat Transf Eng 29:432-460

Zhang X, Gu H, Fujii M (2006) Experimental study on the effective thermal conductivity and thermal diffusivity of nanofluids. Int J Thermophys 27:569-580 